\title{
Ionic Conductance of Polyelectrolyte-Modified Nanochannels: Nanoconfinement Effects on the Coupled Protonation Equilibria of Polyprotic Brushes
}

\author{
Facundo M. Gilles, ${ }^{\dagger}$ Mario Tagliazucchi, ${ }^{*} \neq{\text { Omar Azzaroni, }{ }^{* \dagger}{ }^{\dagger} \text { and Igal Szleifer }}^{\S}$ \\ ${ }^{\dagger}$ Instituto de Investigaciones Fisicoquímicas Teóricas y Aplicadas (INIFTA), CONICET, Departamento de Química, Facultad de \\ Ciencias Exactas, Universidad Nacional de La Plata, La Plata 1900, Argentina \\ ${ }^{\ddagger}$ INQUIMAE-CONICET, Ciudad Universitaria, Pabellón 2, and Ciudad Autónoma de Buenos Aires, Buenos Aires C1428EHA, \\ Argentina \\ ${ }^{\S}$ Department of Biomedical Engineering, Department of Chemistry and Chemistry of Life Processes Institute, Northwestern \\ University, Evanston, Illinois 60208, United States
}

\section{Supporting Information}

ABSTRACT: A theoretical methodology is introduced to calculate the low-bias conductance, structure, and composition of long polyelectrolyte-modified nanochannels of arbitrary geometry. This methodology is applied to explore the coupling between acid-base equilibrium and geometry in cylindrical, conical, and trumpet-shaped nanochannels modified by end-grafted layers of poly(2(methacryloyloxy)ethyl-phosphate) (PMEP), a diprotic polyacid. The ionic conductance and speciation curves (i.e., the fraction of deprotonated, monoprotonated, and diprotonated acid segments) for this system were predicted as a function of the solution $\mathrm{pH}$. The apparent $\mathrm{p} K_{\mathrm{a}}^{\prime}$ 's and widths of the transitions between the different acid-base states determined from the speciation curves depend on the diameter and shape of the nanochannel and the bulk salt concentration. In the limit of wide channels, the apparent $\mathrm{p} K_{\mathrm{a}}$ 's and widths can be

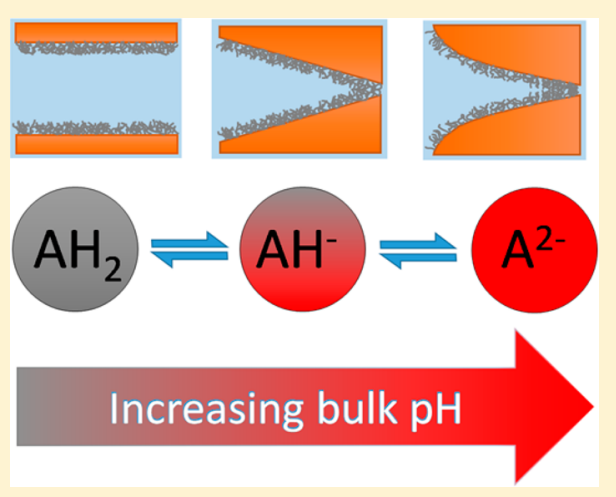
estimated by a simplified analytical model derived from the more general molecular theory. Both the general and the simplified theory predicts that, due to charge-regulation effects, the first acid-base transition $(0 /-1$ transition) is wider than the second one $(-1 /-2)$, and both transitions are wider than the ideal one expected for an isolated acid-base group in the bulk. It is also shown that the inflection points of the conductance versus $\mathrm{pH}$ curves provide a very good estimation of the apparent $\mathrm{p} K_{\mathrm{a}}$ 's of the polyelectrolyte for cylindrical channels, but the quality of the estimation decreases for noncylindrical geometries.

\section{INTRODUCTION}

In the last 15 years, the study of solid-state nanochannels has led to the discovery of novel physical and chemical phenomena, such as current rectification and surface-governed unipolar ion transport. ${ }^{1-7}$ The typical diameters of nanochannels range from $\sim 10$ to $\sim 100 \mathrm{~nm}$; therefore, they exhibit a high surface-tovolume ratio, which allows manipulation of ion fluxes through the properties of the inner wall. Inspired by biological nanochannels, ${ }^{1,3,5,8-11}$ researchers have explored theoretically and experimentally the role of the shape ${ }^{12,13}$ and the surface charge $^{14-16}$ of the nanochannels on their ionic conductance. These studies had led to potential applications in diverse fields, including energy conversion, ${ }^{1,17,18}$ ionic logic circuits, ${ }^{19-21}$ and biological sensors. ${ }^{4,5,22-27}$ Each of these applications has different requirements in terms of current-potential behavior and response to stimuli, and thus applications require a deep understanding of the effects of the geometry of the channel and the chemical properties of the channel's inner wall on the ionic conductance. The present study reports a new theoretical methodology to calculate the low-bias conductance of nano- channels of arbitrary shape as well as new theoretical insights to understand the coupled effects of channel geometry and surface chemistry on conductance. We specifically analyze a model system of a nanochannel modified by end-grafted poly-2(methacryloyloxy)ethyl phosphate (PMEP) polyelectrolytes. We chose this system because PMEP is a diprotic polyacid, which allows us to characterize how the two different acid-base equilibria of PMEP and the coupling between them are modulated by nanoconfinement effects imposed by nanochannels of varying dimensions and geometry. Moreover, nanochannels ${ }^{28}$ and nanopores ${ }^{29}$ modified by PMEP have been studied experimentally and are interesting candidates for the construction of synthetic hybrid assemblies displaying properties observed in biological ion channels.

Grafting weak polyelectrolytes to the inner wall of a nanochannel creates a responsive system where the number

Received: December 2, 2015

Revised: February 11, 2016 
of chargeable monomers increases with the degree of polymerization and the state of charge can be controlled by the $\mathrm{pH}$ and ionic strength of the solution. Nanoconfinement by the nanochannel enhances the coupling between physical interactions, chemical equilibria, and molecular organization, as shown in previous experimental and theoretical work. ${ }^{28-31}$ In this context, confinement emerges as a potential strategy to modulate the kinetics and thermodynamics of chemical reactions in nanoscale systems. However, it is very challenging to measure and control chemical reactions in confined environments; for example, the state of protonation of a weak polyelectrolyte within a single nanochannel cannot be directly accessed from experiments, and thus it has to be inferred from conductance experiments.

We combine here an established molecular theory to treat chemical equilibrium in confined environments with a new approach to determine the low-bias conductance of polyelectrolyte-modified long nanochannels of arbitrary shapes. We show that the apparent chemical equilibrium constants $\left(\mathrm{p} K_{\mathrm{a}}\right)$ of the diprotic polyacid inside the channel are shifted with respect to the $\mathrm{p} K_{\mathrm{a}}$ 's of the monomers in homogeneous solution and depend on the shape of the channel. Furthermore, the $\mathrm{pH}$ ranges where the acid-base transitions occur are wider than those in bulk solution, and the first acid-base transition is always wider than the second. We show that the conductance versus $\mathrm{pH}$ curve allows quantitative determination of the apparent $\mathrm{p} K_{\mathrm{a}}$ of the polyacid inside the nanochannel for a cylindrical geometry and an approximate determination for other geometries, which validates the use of the $\mathrm{pH}$ dependence of the channel conductance to infer the protonation states of nanoconfined species.

\section{THEORETICAL METHOD}

The system under study, schematized in Figures 1 and 2, consists of a single nanochannel modified by PMEP chains end-

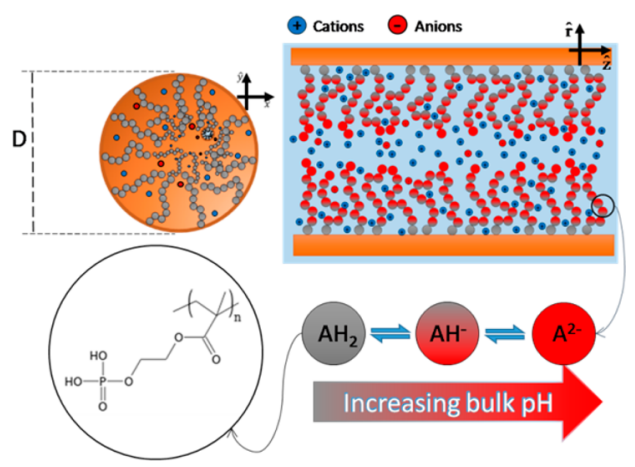

Figure 1. Scheme of a section of cylindrical nanochannel modified by an end-grafted layer of the weak polyacid (poly(2-(methacryloyloxy)ethyl-phosphate)), PMEP. The solution inside the nanochannel contains water molecules, salt anions (small red circles), salt cations (small blue circles), hydroxyl ions, and protons. Each monomer in PMEP can exist in one of three possible chemical states: $\mathrm{PMEPH}_{2}$ $\left(\mathrm{AH}_{2}\right), \mathrm{PMEPH}^{-}\left(\mathrm{AH}^{-}\right)$, and $\mathrm{PMEP}^{2-}\left(\mathrm{A}^{2-}\right)$.

grafted to the inner surface. We will consider in this work cylindrical, conical, or trumped-shaped nanochannels, although our methodology can be applied to an arbitrary geometry. The channel connects two macroscopic reservoirs containing identical aqueous solutions of $\mathrm{KCl}$ at a fixed concentration and $\mathrm{pH}$. We will explore the connection between the shape, structure, and composition of these channels and its macro-
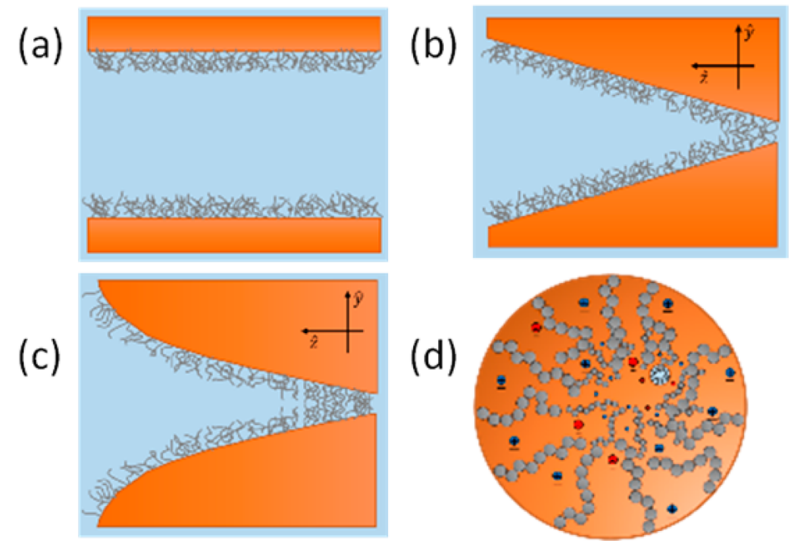

Figure 2. Panels (a), (b), and (c) show schemes of the geometries explored (not in scale). Locally each geometry can be approximated as cylinder due to the large aspect ratio of the channel. Panel (d) shows a transversal view of the channels. We assume cylindrical symmetry, meaning that inhomogeneities are considered in the radial $(r)$ and principal axis directions $(z)$ only.

scopic conductance with a new modeling approach based on a previously reported molecular theory. ${ }^{28,31,33,34}$ We start by formulating the molecular theory for a cylindrical channel at equilibrium. The molecular theory explicitly considers the shape, size, charge, and conformations of all of the molecular species of the system and the acid-base equilibria of the segments of the tethered weak polyelectrolyte. The theoretical approach consists of writing the free energy of the system as a functional of the densities of the mobile species (water molecules, $\mathrm{Cl}^{-}, \mathrm{K}^{+}, \mathrm{H}^{+}$, and $\mathrm{OH}^{-}$), the probability distribution function of the polyelectrolyte conformations, and the positiondependent fractions of the chemical states of the monomers. These functions are then obtained by finding the extremum of the free energy functional. We propose a free energy that has the following contributions: conformational entropy of the polyelectrolyte $S_{\text {pol }}$ mixing entropy $S_{\text {mix }}$ of the free ions $\left(\mathrm{K}^{+}\right.$, $\mathrm{Cl}^{-}, \mathrm{OH}^{-}$, and $\mathrm{H}^{+}$) and water molecules $(\mathrm{w})$, the chemical free energy $F_{\mu}$ associated with the acid-base equilibrium of PMEP segments, and the electrostatic energy $F_{\text {elec }}$ :

$$
F=-T S_{\text {pol }}-T S_{\text {mix }}+F_{\mu}+F_{\text {elec }}
$$

In this work, we will consider good solvent conditions, and therefore we do not include the contribution due to attractive van der Waals (vdW) interactions. ${ }^{35}$

The system is in contact with a bath (solution) of all mobile species, and therefore we use a semigrand canonical ensemble, where the number of ions and solvent molecules inside the nanochannel is determined by the condition of constant chemical potential everywhere in the system. The semigrand potential is the Legendre transform of the Helmholtz free energy. For a cylindrical nanochannel, the semigrand potential per unit area is given by

$$
\beta W=\frac{\beta F}{2 \pi R L}-\sum_{i} \beta \mu_{i} N_{i}
$$

where $\mu_{i}$ and $N_{i}$ are the chemical potential and total number of molecules of species $i$ per unit area, respectively, $R$ is the channel radius, $L$ is the channel length, $\beta=1 / k_{\mathrm{B}} T, k_{\mathrm{B}}$ is the Boltzmann constant, $T$ is the temperature, and the sum runs over all of the free species. We add to the semigrand canonical free energy two constraints to account for the global 
electroneutrality and the local incompressibility (packing constraint). The detailed expression of the thermodynamic potential per unit area is

$$
\begin{aligned}
& \beta \Theta=\sigma \sum_{\alpha} P(\alpha) \ln (P(\alpha)) \\
& +\sum_{i=\mathrm{K}^{+}, \mathrm{Cl}^{-}, \mathrm{H}^{+}, \mathrm{OH}^{-}, \mathrm{w}} \int_{0}^{R} \mathrm{~d} r \frac{r}{R} \rho_{i}(r)\left[\ln \left(\rho_{i}(r) v_{\mathrm{w}}\right)-1+\beta \mu_{i}^{\mathrm{o}}\right] \\
& +\int_{0}^{R} \mathrm{~d} r \frac{r}{R}\left\langle\rho_{p}(r)\right\rangle\left\{\sum_{e=0}^{2} f_{e}(r)\left[\ln \left(f_{e}(r)\right)+\beta \mu_{e}^{o}\right]\right\} \\
& +\beta \int_{0}^{R} \mathrm{~d} r \frac{r}{R}\left[\left\langle\rho_{q}(r)\right\rangle \beta \psi(r)-\frac{1}{2} \beta \varepsilon\left(\nabla_{r} \psi(r)\right)^{2}\right] \\
& -\sum_{j=\mathrm{K}^{+}, \mathrm{Cl}^{-}, \mathrm{OH}^{-}, \mathrm{w}} \beta \mu_{j} \int_{0}^{R} \mathrm{~d} r \frac{r}{R} \rho_{j}(r) \\
& +\lambda \int_{0}^{R} \mathrm{~d} r \frac{r}{R}\left\langle\rho_{q}(r)\right\rangle \\
& +\int_{0}^{R} \mathrm{~d} r \frac{r}{R} \beta \pi(r)\left[\left\langle\phi_{p}(r)\right\rangle+\sum_{i=\mathrm{K}^{+}, \mathrm{Cl}^{-}, \mathrm{H}^{+}, \mathrm{OH}^{-}, \mathrm{w}} \phi_{i}(r)-1\right] \\
& -\beta \mu_{\mathrm{H}^{+}} \int_{0}^{R} \mathrm{~d} r \frac{r}{R}\left[\rho_{\mathrm{H}^{+}}(r)+\left(2 f_{0}(r)+f_{-1}(r)\right)\left\langle\rho_{p}(r)\right\rangle\right]
\end{aligned}
$$

The first term in eq 3 corresponds to the conformational entropy of the tethered polymers; $\sigma$ is the grafting density of PMEP defined as the number of chains per area, and $P(\alpha)$ is the probability of having a chain in conformation $\alpha$. The second term corresponds to the mixing (translational) entropy of the solvent and free ions and the standard chemical potential $\left(\mu_{i}^{\mathrm{o}}\right)$ contribution for these species. In this term, $\rho_{i}(r)$ is the number density at $r, r$ is the radial coordinate, and $v_{i}$ is the molecular volume of species $i=\mathrm{K}^{+}, \mathrm{Cl}^{-}, \mathrm{H}^{+}, \mathrm{OH}^{-}$, w.

The third term is the free energy associated with the acidbase chemical equilibria:

$$
\mathrm{AH}_{2} \stackrel{K_{\mathrm{a} 1}}{\rightleftarrows} \mathrm{AH}^{-}+\mathrm{H}^{+}, \quad \mathrm{AH}^{-} \stackrel{K_{\mathrm{a} 2}}{\rightleftharpoons} \mathrm{A}^{2-}+\mathrm{H}^{+}
$$

and represents the mixing entropy between segments in different chemical states. The polymer volume fraction $\left\langle\phi_{p}(r)\right\rangle$ is the product of the polymer monomer number density, $\left\langle\rho_{p}(r)\right\rangle$, and the volume of a monomer, $v_{p}$. The monomers in the polymer can have zero, one, or two negative charges, which correspond to $e=0,-1,-2$. The fraction of monomers in state $e$ is $f_{e}(r)$, and $\mu_{e}^{\mathrm{o}}$ is the standard chemical potential of the monomer when its charge is $e$. The fractions of monomers are normalized so that $\sum_{e}^{2}={ }_{0} f_{e}(r)=1$.

For free monomers in bulk solution, the concentration of monomers in each chemical state is determined by two equilibrium constants:

$$
K_{\mathrm{a} 1}=\frac{\left[\mathrm{AH}^{-}\right]\left[\mathrm{H}^{+}\right]}{\left[\mathrm{AH}_{2}\right]}, \quad K_{\mathrm{a} 2}=\frac{\left[\mathrm{A}^{2-}\right]\left[\mathrm{H}^{+}\right]}{\left[\mathrm{AH}^{-}\right]}
$$

where $[j]$ denotes the molar concentration of species $j$ (we approximated activities by molar concentrations in these expressions). The standard free energy of the reactions $\Delta G_{1}^{0}$ $=\mu_{-1}^{0}+\mu_{\mathrm{H}^{+}}^{0}-\mu_{0}^{0}$ and $\Delta G_{2}^{0}=\mu_{-2}^{0}+\mu_{\mathrm{H}^{+}}^{0}-\mu_{-1}^{0}$, and the equilibrium constants are related by $K_{\mathrm{a} i}=C \exp \left(-\beta \Delta G_{\mathrm{i}}^{0}\right)$ (with $i=1,2)$, where $C=\rho_{\mathrm{w}}^{\text {bulk }} / N_{\mathrm{A}}$ is a constant necessary for consistency of units.
The fourth term in eq 3 represents the electrostatic energy, where $\psi(r)$ is the local electrostatic potential at position $r$, $\left\langle\rho_{q}(r)\right\rangle$ is the local mean density of charge at $r$, and $\varepsilon$ is the dielectric constant of the solution inside the nanochannel. The density of charge is given by

$$
\begin{aligned}
& \left\langle\rho_{q}(r)\right\rangle=-\left\langle\rho_{p}(r)\right\rangle\left(f_{-1}(r)+2 f_{-2}(r)\right) \\
& +\sum_{i=\mathrm{K}^{+}, \mathrm{Cl}^{-}, \mathrm{H}^{+}, \mathrm{OH}^{-}} \rho_{i}(r) q_{i}
\end{aligned}
$$

where $q_{i}$ represents the charge of each free ion species.

The fifth term enforces constant chemical potentials across the system for $\mathrm{K}^{+}, \mathrm{Cl}^{-}, \mathrm{OH}^{-}$, and $\mathrm{w}$. The sixth term is the global electroneutrality constraint, enforced by the Lagrange multiplier $\lambda$. The seventh term is the local incompressibility constraint, which models the intermolecular repulsive interactions as a packing constraint. The repulsive interactions are considered as excluded volume interactions that are accounted for through the constraint that the available volume element at $r$ is exactly the sum of the volume filled by the each of the different chemical species at $r$, that is:

$$
\phi_{\mathrm{w}}(r)+\left\langle\phi_{p}(r)\right\rangle+\sum_{i=\mathrm{K}^{+}, \mathrm{Cl}^{-}, \mathrm{H}^{+}, \mathrm{OH}^{-}} \phi_{i}(r)=1
$$

where $\phi_{i}(r)$ is the volume fraction of species $i$ at $r$. This constraint is enforced in the free energy expression, eq 3, through the Lagrange multipliers $\pi(r)$, which correspond to local osmotic pressures.

The position-dependent density of each molecular species $\rho_{i}(r)$, the polymer probability distribution $P(\alpha)$, the different degrees of dissociation $f_{e}(r)$, the electrostatic potential $\psi(r)$, and the local osmotic pressure $\pi(r)$ inside the nanochannel are obtained in equilibrium conditions through minimization of the semigrand canonical potential, eq 3 .

The minimization of the semigrand canonical functional with respect to the densities of mobile ions and solvent yields:

$$
\begin{gathered}
\phi_{i}(r)=\exp \left[\beta \mu_{i}-\beta \pi(r) v_{i}-\beta \psi(r) q_{i}\right], \\
i=\mathrm{Cl}^{-}, \mathrm{K}^{+}, \mathrm{OH}^{-}, \mathrm{H}^{+}, \mathrm{w}
\end{gathered}
$$

This expression shows the functional dependence of the position-dependent volume fraction of species $i$ inside the nanochannel with the local osmotic pressure and the local electrostatic potential.

For the degree of charge, we obtain

$$
\begin{aligned}
& f_{-1}(r)=\frac{K_{1} \phi_{\mathrm{H}^{+}}^{b} \exp [-\beta \psi(r)|e|]}{\left(\phi_{\mathrm{H}^{+}}^{b} \exp [-\beta \psi(r)|e|]\right)^{2}+K_{1}\left(\phi_{\mathrm{H}^{+}}^{b} \exp [-\beta \psi(r)|e|]\right)+K_{1} K_{2}} \\
& f_{-2}(r)=\frac{K_{1} K_{2}}{\left(\phi_{\mathrm{H}^{+}}^{b} \exp [-\beta \psi(r)|e|]\right)^{2}+K_{1}\left(\phi_{\mathrm{H}^{+}}^{b} \exp [-\beta \psi(r)|e|]\right)+K_{1} K_{2}}
\end{aligned}
$$

where $|e|$ is the elementary charge. These equations demonstrate how the local degree of protonation depends on the bulk $\mathrm{pH}$, through the bulk concentration of protons, and the local electrostatic potential. It is important to emphasize that the local electrostatic potential depends on the distribution of all charged species in the system, and, therefore, the degree of protonation depends on the overall molecular organization within the pore. In the bulk, $\psi=0$, and thus eqs 9 and 10 reduce to the bulk relationships between degrees of 
protonation and acid-base equilibrium constants for diprotic acids. $^{36}$

Minimization of the free energy functional with respect to $P(\alpha)$ yields the following expression for the probability distribution of the polyelectrolyte:

$$
\begin{aligned}
P(\alpha)= & \frac{1}{Q} \exp \left\{-\int_{0}^{R} \mathrm{~d} r \frac{r}{R} n_{p}(r, \alpha)\left[\beta v_{p} \pi(r)\right.\right. \\
& \left.\left.+\ln \left(f_{0}(r)\right)\right]\right\}
\end{aligned}
$$

where $n_{p}(r, \alpha)$ is the number of segments that a chain in conformation $\alpha$ has in the cylindrical shell between $r$ and $r+\mathrm{d} r$, and $Q$ assures normalization of $P(\alpha)$.

The extremum of the free energy functional with respect to the electrostatic potential leads to the Poisson equation of the form:

$$
\nabla^{2} \psi(r)=-\frac{\left\langle\rho_{q}(r)\right\rangle}{\varepsilon}
$$

The equilibrium properties of the polyelectrolyte-modified nanopore are obtained by numerically solving the system of equations given by the Poisson equation, eq 12, the packing constraint, eq 7 , the position-dependent densities of mobile species, eq 8, the position-dependent fractions of the chemical states of the monomers, eqs 9 and 10, and the probability distribution function of the polyelectrolyte chains, eq 11 (for details, see the Supporting Information). From the numerical solution of the theory, thermodynamic and equilibrium structural properties for the cylindrical channel can be calculated, including the distribution of ions within the pore as well as the position-dependent state of ionization of the monomers.

\section{CONDUCTANCE OF NANOCHANNELS WITH CYLINDRICAL AND NONCYLINDRICAL GEOMETRIES}

To describe different shapes of channels, we use the following analytical expression for the change of the channel diameter, $D$, along the channel main axis, $z$ :

$$
D(z)=\left(D_{\max }-D_{\min }\right)\left(\frac{z}{L}\right)^{n}+D_{\min }
$$

Note that $n=0,1$, and 2 correspond to cylindrical, conical, and trumpet-shaped channels (see Figure 2) with apertures of diameter $D_{\min }$ (tip) and $D_{\max }$ (base). While eq 13 is a convenient analytical expression to describe the shape of the nanochannel, the theory described below is not limited to shapes described by this expression; that is, our theory can be used to model arbitrary shapes as long as the nanochannel has a large aspect ratio.

The main idea behind our theoretical approach to model the conductance of a long nanochannel with arbitrary shape is to split it into approximately cylindrical slices and approximate its total resistance as the series combination of the resistances of these slices. In other words, we approximate the total resistance of the nanochannel as the sum of the resistances of $N=L / d z$ segments of length $\mathrm{d} z$ and diameter $D(z)$. This approximation is valid because the length of the nanochannel $(L \approx 12 \mu \mathrm{m})$ is much larger than its diameter $(D \approx 100 \mathrm{~nm})$, and, therefore, the channel can be locally approximated as a cylinder of diameter $D(z)$. In the case where $D \approx L$, this approximation breaks down and a fully two-dimensional calculation is required; see, for example, ref 37. Let us define $\omega(z) \mathrm{d} z$ as the resistance of a cylindrical slice of diameter $D(z)$ (note that $\omega(z)$ has units of $\Omega \mathrm{m}^{-1}$ ). In the low-bias limit (i.e., vanishing applied bias), we can calculate the total resistance of the nanochannel, $\Omega^{\text {tot }}$, as

$$
\Omega^{\mathrm{tot}}=\int_{0}^{L} \omega(z) \mathrm{d} z
$$

The differential of length $\mathrm{d} z$ can be transformed into a differential of channel diameter $\mathrm{d} D$ by differentiation of both sides of eq 13 , which yields

$$
\mathrm{d} z=\frac{L}{n}\left(\frac{z}{L}\right)^{1-n} \frac{\mathrm{d} D}{\left(D_{\max }-D_{\min }\right)}
$$

Replacing eqs 13 and 15 with eq 14 finally yields

$$
\Omega^{\mathrm{tot},(n)}=\frac{L}{n} \frac{1}{\left(D_{\max }-D_{\min }\right)^{1 / n}} \int_{D_{\min }}^{D_{\max }} \frac{\omega(D)}{\left(D-D_{\min }\right)^{1-1 / n}} \mathrm{~d} D
$$

Equation 16 allows us to calculate the low-bias resistance $\Omega^{\text {tot, }(n)}$ and conductance, $G^{(n)}=1 / \Omega^{\text {tot, }(n)}$, of noncylindrical long nanochannels modified by polyelectrolytes from the knowledge of the resistance of a cylindrical channel of diameter $D$ and thickness $\mathrm{d} z$. This resistance can be obtained from the molecular theory described above using the Nernst-Planck equation under the Goldman constant-field approximation: ${ }^{38}$

$$
\omega(D)=\left(2 \pi \frac{F^{2}}{R T} \sum_{i=\mathrm{A}, \mathrm{C}, \mathrm{H}^{+}, \mathrm{OH}^{-}} q_{i}^{2} \bar{D}_{i} \int_{0}^{D / 2} c_{i}(r) r \mathrm{~d} r\right)^{-1}
$$

where $F$ is the Faraday constant, $R$ is the gas molar constant, $D$ is the channel diameter, $\bar{D}_{i}$ is the diffusion coefficient of ion $i$ (which we approximate to that in water), and $c_{i}(r)$ is the molar concentration of ion $i$ inside the cylindrical section predicted by the molecular theory.

In summary, the model outlined above allows us to calculate the low-bias conductance of polyelectrolyte-modified long nanochannels of arbitrary shape. We tested the approximation of modeling a noncylindrical channel as the combination of cylindrical slices of different diameters for the case of a conical nanochannel with constant surface charge (i.e., without the presence of grafted polyelectrolytes). We chose this system because it can be modeled using the Nernst-Planck-Poisson equations and solved without further approximations using finite elements (FE) calculations. Figure 3a shows that the conductance predicted by our theoretical approach is in excellent agreement with FE calculations that explicitly modeled the complete channel.

Our theory can be also used to successfully fit the available experimental conductance versus $\mathrm{pH}$ curves for PMEPmodified conical nanochannels; ${ }^{39}$ see Figure $3 \mathrm{~b}$. The black points in the figure correspond to the experimental low-bias conductance, which was determined as the slope of the $I-V$ curve for $V \rightarrow 0$ using the raw data plotted in Figure 3a of ref 39. To fit the experimental data, we fixed the nanochannel dimensions to the values reported in ref 39 . In the calculation, we fixed the dimensions of the channel $\left(D_{\min }=16 \mathrm{~nm}, D_{\max }=\right.$ $290 \mathrm{~nm}$, and $L=12 \mu \mathrm{m})$, the bulk $\mathrm{p} K_{\mathrm{a}}$ constants of PMEP, and the bulk salt concentration $\left(C_{\text {salt }}=0.1 \mathrm{M}\right)$, and we allowed the degree of polymerization $N$ and the surface coverage $\sigma$ to vary 

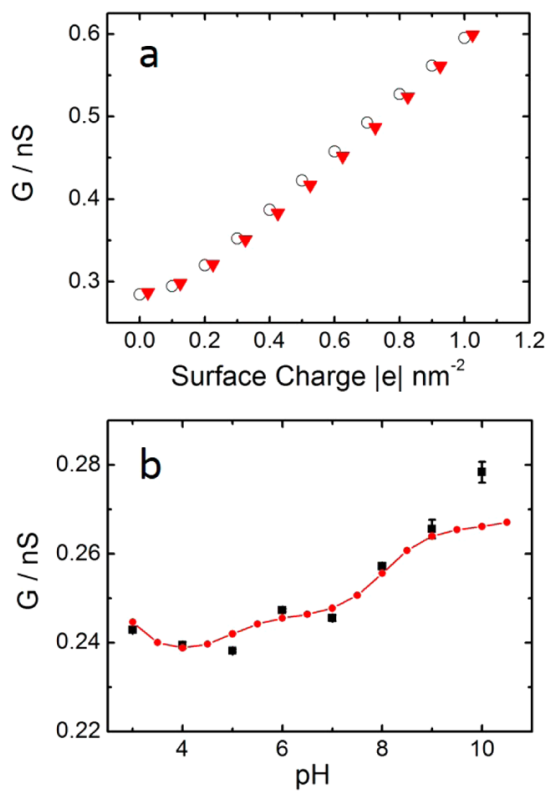

Figure 3. (a) Comparison of the conductance of a conical nanochannel $\left(D_{\min }=10 \mathrm{~nm}, D_{\max }=290 \mathrm{~nm}\right.$, and $\left.L=12 \mu \mathrm{m}\right)$ as a function of the surface charge. Circles show the conductance obtained with our theoretical methodology, and triangles show the solution of the Nernst-Planck-Poisson equations using finite element calculations, respectively. For details on the finite elements calculations, see the Supporting Information of ref 40. (b) Experimental conductance versus $\mathrm{pH}$ results for PMEP-modified nanochannels (ם) and theoretical fit. The experimental conductances were extracted from the $I$ versus $V$ data at $V \rightarrow 0$ for a PMEP-modified nanochannel (data published in Figure 3a of ref 39; the error represents the errors associated with finding the slope of the plot around $V=0$ ).

(we used $N=14$ and $\sigma=0.01$ chains $\mathrm{nm}^{-2}$; in practice, the relevant parameter is $N \cdot \sigma,{ }^{28}$ so other combinations of $N$ and $\sigma$ are possible). We also allowed the geometry to slightly depart from an ideal conical shape: we used $n=1.3$, which is close to the ideal conical shape $(n=1)$. The fact that our theoretical methodology agrees very well with less approximate theoretical calculations (Figure 3a) and that it can be successfully used to fit experimental results (Figure $3 b$ ) supports its validity as a tool to study the conductance of long cylindrical and noncylindrical nanochannels.

\section{RESULTS AND DISCUSSION}

Acid-Base Equilibria and Conductance in Cylindrical Nanochannels. Figure 4 shows predictions for a polyelectrolyte-modified cylindrical nanochannel with diameter $D=16$ $\mathrm{nm}$ and bulk salt concentration $C_{\text {salt }}=0.1 \mathrm{M}$. Figure 4a shows the $\mathrm{pH}$ dependence of the degree of dissociation $\left\langle f_{e}\right\rangle$, for $e=0$, -1 , and -2 , where $e$ denotes one of the three possible states of charge of the MEP monomers in PMEP (see Figure 1). These states correspond to the phosphoric acid $\left(\mathrm{AH}_{2}=\mathrm{R}-\mathrm{PO}_{4} \mathrm{H}_{2}, e\right.$ $=0)$, the monovalent charged $\left(\mathrm{AH}^{-}=\mathrm{R}-\mathrm{PO}_{4} \mathrm{H}^{-}, e=-1\right)$, and the divalent charged phosphate $\left(\mathrm{A}^{2-}=\mathrm{R}-\mathrm{PO}_{4}{ }^{2-}, e=-2\right)$ species. The chemical equilibrium constants for the phosphate groups in the bulk (see eqs 4 and 5) are $\mathrm{p} K_{\mathrm{a} 1}^{\text {bulk }}=4.5$ and $\mathrm{p} K_{\mathrm{a} 2}^{\text {bulk }}=7.7$. These bulk values do not consider the effect of the local environment on the acid-base equilibrium. The molecular theory incorporates local environment effects into the bulk equilibrium constants, which leads to apparent equilibrium
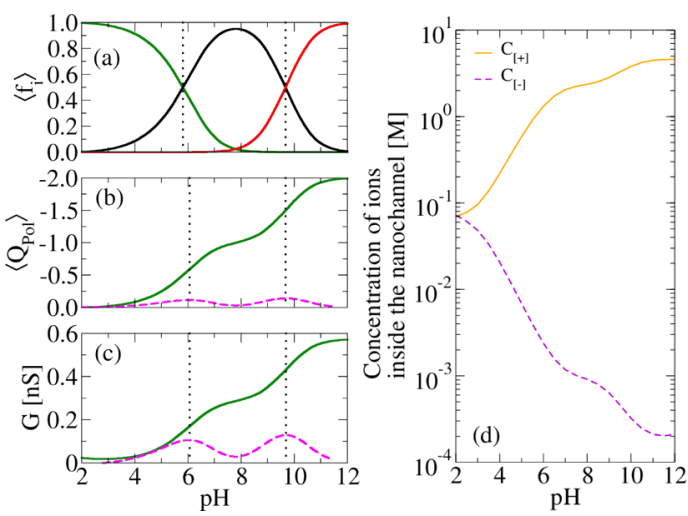

Figure 4. Cylindrical nanochannel behavior when PMEP is endgrafted to the inner surface. $\mathrm{p} K_{\mathrm{a} 1}^{\text {bulk }}=4.5, \mathrm{p} K_{\mathrm{a} 2}^{\text {bulk }}=7.7$. (a) Average degree of dissociation. The fractions of monomers in uncharged state $\left\langle f_{0}\right\rangle$, the single charged state $\left\langle f_{-1}\right\rangle$, and the double charged state $\left\langle f_{-2}\right\rangle$ are shown in green, black, and red lines, respectively. The apparent $\mathrm{p} K_{\mathrm{a}}$ 's $\left(\mathrm{p} K_{\mathrm{a}}^{\mathrm{a} p \mathrm{p}}\right)$ were determined from these curves as the $\mathrm{pH}$ 's where $\left\langle f_{0}\right\rangle=\left\langle f_{-1}\right\rangle\left(\mathrm{p} K_{\mathrm{a} 1}^{\mathrm{app}}=5.81\right)$ and $\left\langle f_{-1}\right\rangle=\left\langle f_{-2}\right\rangle\left(\mathrm{p} K_{\mathrm{a} 2}^{\mathrm{app}}=9.67\right)$. (b) Mean charge per monomer as a function of $\mathrm{pH}$ (green solid line) and its first derivative with respect to $\mathrm{pH}$ (magenta dashed line). (c) Channel conductance (green solid line) and its first derivative with respect to $\mathrm{pH}$ (magenta dashed line). The apparent $\mathrm{p} K_{\mathrm{a}}$ 's estimated from this plot as the maximum of the first derivative are $\mathrm{p} K_{\mathrm{a} 1}^{\text {cond }}=6.06$ and $\mathrm{p} K_{\mathrm{a} 2}^{\text {cond }}=9.69$. (d) Average concentration of anions and cations inside the nanochannel as a function of solution $\mathrm{pH}$. Calculation parameters: $D=16 \mathrm{~nm}, C_{\text {salt }}=0.1 \mathrm{M}, N=28$ units per chain, $N_{\mathrm{p}} / A(R)$ $=0.2$ chains $/ \mathrm{nm}^{2}$.

constants within the nanochannels that are different from the bulk ones, as we discuss next.

The first chemical equilibrium involves the transition from the uncharged state (at $\mathrm{pH}=2.0$ ) to the single charged state (at $\mathrm{pH} \approx 8.0$ ), with an almost zero population of monomers in the state of charge $e=-2$. For $\mathrm{pH}$ values greater than 8 , the fraction of uncharged monomers is almost zero $\left(\left\langle f_{0}\right\rangle \approx 0\right)$, and the number of monomers in state -2 increases at the expense of monomers in the state -1 . For $\mathrm{pH}>12$, the PMEP behaves as a strong polyelectrolyte with all of the groups in the charge state -2 . The average charge per monomer $\left\langle Q_{\text {mon }}\right\rangle=-\left\langle f_{1}\right\rangle|e|-$ $2\left\langle f_{2}\right\rangle \mid e l$, shown in Figure $4 \mathrm{~b}$, increases in absolute value with increasing $\mathrm{pH}$ and reaches $-2 \mid \mathrm{l} l$ at $\mathrm{pH} \approx 12$. The conductance of the system $(G)$ in Figure $4 c$ increases with increasing solution $\mathrm{pH}$. This effect is due to the increase of the total concentration of cation counterions inside the channel that are needed to compensate the increasing charge of the polyelectrolyte. These counterions act as free charge carriers; therefore, the conductance of the nanochannel increases according eq 14. At $\mathrm{pH}=12$, the nanochannel is highly permselective, and the concentration of counterions is predicted to be 4 orders of magnitude larger than the concentration of co-ions; see Figure $4 \mathrm{~d}$. Because of this asymmetric concentration of free ions, the solution inside the nanochannel is referred to as a "unipolar" solution. ${ }^{18}$

The values of apparent $\mathrm{p} K_{\mathrm{a}}$ 's of confined polyelectrolytes are different from the bulk $\mathrm{p} K_{\mathrm{a}}$ 's values due to local environment effects; see, for example, ref 28 . We define the first and the second apparent chemical equilibrium constants as the $\mathrm{pH}$ values where $\left\langle f_{0}\right\rangle=\left\langle f_{-1}\right\rangle$ and $\left\langle f_{-1}\right\rangle=\left\langle f_{-2}\right\rangle$, respectively. Note that this is the usual definition of apparent $\mathrm{p} K_{\mathrm{a}}$; that is, the apparent $\mathrm{p} K_{\mathrm{a}}$ is defined as the $\mathrm{pH}$ value where the fractions of the two chemical states involved in the chemical equilibrium are 
the same. We find $\mathrm{p} K_{\mathrm{al}}^{\mathrm{app}}=5.81$ and $\mathrm{p} K_{\mathrm{a} 2}^{\mathrm{app}}=9.67$ for the system in Figure $4\left(N=28\right.$ monomers per chain, $N_{\mathrm{p}} / A(R)=$ 0.2 chains $\left./ \mathrm{nm}^{2}, D=16 \mathrm{~nm}, C_{\text {salt }}=0.1 \mathrm{M}\right)$. Note that the determination of $\mathrm{p} K_{\mathrm{al}}^{\mathrm{app}}$ requires knowledge of the average degrees of dissociation, which cannot be experimentally measured in a single nanochannel, to calculate the apparent equilibrium constants. On the other hand, the conductance of the nanochannel is a experimentally measurable quantity that is routinely used to estimate the state of charge of the polyelectrolyte. $^{28}$ To study the accuracy of estimating the apparent $\mathrm{p} K_{\mathrm{a}}$ 's from conductance data, let us define the chemical equilibrium constants $\mathrm{pK}_{\mathrm{a} 1}^{\text {cond }}$ and $\mathrm{p} K_{\mathrm{a} 2}^{\text {cond }}$ as the $\mathrm{pH}$ values where the first derivative of the conductance reaches a maximum, that is, the inflection points of the $G$ versus $\mathrm{pH}$ curves (the superindex "cond" denotes apparent equilibrium constants estimated from the conductance). The vertical dotted lines in Figure $4 \mathrm{c}$ show the apparent $\mathrm{p} K_{\mathrm{a}}$ 's determined from the predicted conductance curves. In the case of the cylindrical nanochannel shown in Figure 4, the apparent equilibrium constants for both acid-base equilibria estimated from the conductance are good estimations of those directly determined from the degrees of dissociation. The value of $\mathrm{p} K_{\mathrm{a} 1}^{\text {cond }}=6.06$ (determined from the conductance) is slightly larger than $\mathrm{pK}_{\mathrm{a} 1}^{\mathrm{app}}=5.81$ (determined from the dissociation fractions) due to the small, but not negligible, contribution of the counterions due to the species $\mathrm{R}-\mathrm{PO}_{4}^{2-}$ to the total conductance near $\mathrm{pH}=$ $\mathrm{pK}_{\mathrm{al}}^{\mathrm{app}}$. In the last section of the paper, we will address noncylindrical channels and explore the effect of geometry on the agreement between these two apparent chemical equilibrium constants.

Polymer Distribution. Figure 5 shows the polymer distribution inside the nanochannel for different $\mathrm{pH}$ 's and

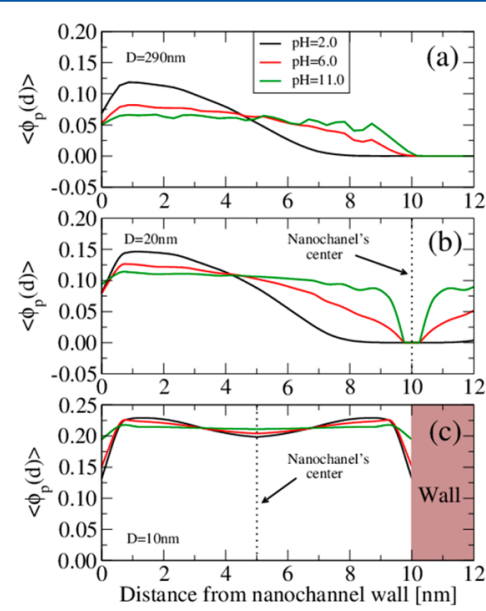

Figure 5. Polymer volume fraction $\left\langle\phi_{\mathrm{p}}(d)\right\rangle$ as a function of distance from the wall of the nanochannel, $d$. The curves correspond to $\mathrm{pH}=$ 2.0 (black), $\mathrm{pH}=6.0$ (red), and $\mathrm{pH}=11.0$ (green). The diameters of the channel are (a) $D=290 \mathrm{~nm}$, (b) $20 \mathrm{~nm}$, and (c) $10 \mathrm{~nm}$. Other parameters: $N=28$ units per chain, $N_{\mathrm{p}} / A(R)=0.2$ chains $/ \mathrm{nm}^{2}, C_{\text {salt }}=$ $0.1 \mathrm{M}$.

states of charge of the polymer: $\mathrm{pH}=2.0$ (uncharged polymer, black curves), $\mathrm{pH}=6.0$ (partially charged, red curves), and $\mathrm{pH}$ $=11.0$ (fully charged, green curves), and different channel diameters $D=290 \mathrm{~nm}$ (panel a), $D=20 \mathrm{~nm}$ (panel b), and $D=$ $10 \mathrm{~nm}$ (panel c).

The fully extended polymer chain has a length of $h \approx 10 \mathrm{~nm}$, which should be compared to the nanochannel radius. When the radius of the channel is greater than or equal to $10 \mathrm{~nm}$ (Figure $5 \mathrm{a}$ for $D=290 \mathrm{~nm}$ and Figure $5 \mathrm{~b}$ for $D=20 \mathrm{~nm}$ ), increasing the $\mathrm{pH}$ swells the polyelectrolyte layer to its maximum thickness of $10 \mathrm{~nm}$ because the polyelectrolyte chains adopt extended conformations upon charging. The coupling between polymer conformation and the state of charge of the monomer is less important for the second chemical equilibrium than for the first one (compare red and green lines in Figure 5a) because before the second deprotonation step, the monomers already have a -1lel charge, and thus the polyelectrolyte chains are already stretched. This result shows that polymer stretching is almost completely saturated after the first deprotonation step. For fully stretched chains on a planar surface (or a very wide channel), the average polymer volume fraction $\left\langle\phi_{p}\right\rangle$ can be estimated by assuming a density profile with a step-function shape, which yields a volume fraction of $\left\langle\phi_{p}\right\rangle \approx N_{p} N v_{p} / A(R) h=0.053$. This result is in agreement with the results in Figure $4 \mathrm{a}$ for a very wide channel, $D=290 \mathrm{~nm}$. However, for a narrow channel with $D=$ $20 \mathrm{~nm}$ (i.e., $h \approx D / 2$ ), we observe $\left\langle\phi_{p}\right\rangle \approx 0.11$ (see green curve in Figure $4 b)$. In this case, the volume fraction of the polymer is larger than that expected on a planar surface due to the fact that for highly curved surfaces the available volume decreases as the distance from the surface increases. Thus, for fixed surface coverage of the polymer, the average polymer density of the layer increases with decreasing $D$.

Figure $5 \mathrm{c}$ shows the volume fraction of polymer inside the nanochannel with diameter $D=10 \mathrm{~nm}$ and different bulk $\mathrm{pH}$ values. In this case, the radius of the channel is smaller than the length of the fully extended polymer; thus $\left\langle\phi_{p}\right\rangle$ is homogeneous within the channel and rather insensitive to the state of charge of the polymer. In this case, $\left\langle\phi_{p}\right\rangle$ is $\sim 0.2$, which is much larger than the value predicted for planar surfaces due to the surface curvature effect explained in the previous paragraph and the overlap of the polymer layers at the center of the channel expected from the fact that $h<D / 2$.

Nanoconfinement Effects on the Chemical Equilibrium. In this section, we focus on the effect of nanoconfinement on the chemical equilibrium constants. For the cylindrical channel of $D=16 \mathrm{~nm}$ and salt concentration $0.1 \mathrm{M}$ (see Figure 4), the theory predicts $\mathrm{p} K_{\mathrm{a} 1}^{\mathrm{app}}=5.81$ and $\mathrm{p} K_{\mathrm{a} 2}^{\mathrm{app}}=9.67$, which are larger than the corresponding bulk equilibrium constants (i.e., the $\mathrm{p} K_{\mathrm{a}}$ for a MEP monomer in the bulk) of $\mathrm{p} K_{\mathrm{al}}^{\text {bulk }}=4.5$ and $\mathrm{p} K_{\mathrm{a} 2}^{\text {bulk }}=7.7$. The shift of the $\mathrm{p} K_{\mathrm{a}}$ equilibrium constants under nanoconfinement emerges from the competition between interactions and molecular organization to minimize the system free energy. As the bulk $\mathrm{pH}$ rises, the polyelectrolyte charges increases, and the electrostatic repulsions among charged monomers increase. The electrostatic repulsions can be reduced by uptake of counterions inside the nanochannel (at the cost of a reduction of the entropy of these counterions), by stretching of polymer chains (at the cost of a reduction of the conformational entropy of these chains), or by shifting the chemical equilibrium toward the uncharged species (at the cost of a higher chemical equilibrium free energy). The third mechanism (shift of chemical equilibrium) implies that, for a polyacid, the apparent $\mathrm{p} K_{\mathrm{a}}$ of the polyacid is larger than that in the bulk solution (bulk $\mathrm{p} K_{\mathrm{a}}$ ). ${ }^{41}$ As shown in previous works, $^{30,31}$ all mechanisms are operational, although polymer stretching is more important during the first protonation than during the second one; compare the polymer distributions at $\mathrm{pH}=2.0$ and $\mathrm{pH}=6.0$ in Figure $4 \mathrm{a}$. 
Confined chemical equilibria also show wider transitions than in the bulk; therefore, for a complete description of chemical equilibrium in confined environments, not only the apparent $\mathrm{p} K_{\mathrm{a}}$ but also the width of the transition should be considered. In Figure 6, we report the effect of the diameter of
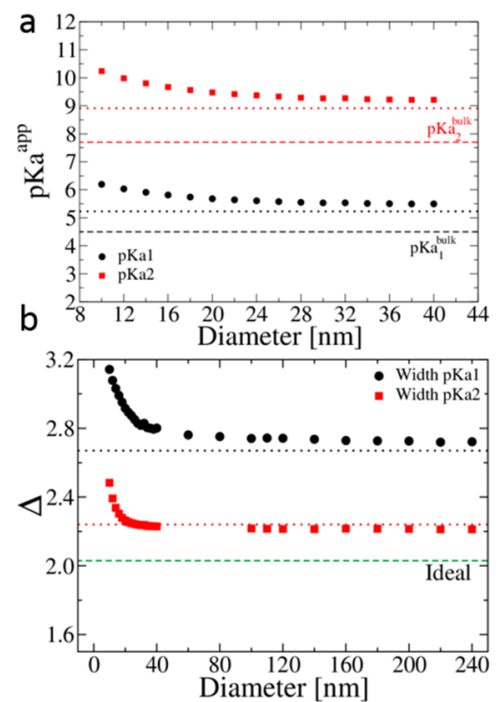

Figure 6. (a) $\mathrm{p} K_{\mathrm{a}}^{\mathrm{app}}$ as a function of cylindrical nanochannel diameter, $D$. The $\mathrm{p} K_{\mathrm{al}}^{\mathrm{app}}$ is defined as the $\mathrm{pH}$ value where $\left\langle f_{0}\right\rangle=\left\langle f_{-1}\right\rangle$, and the $\mathrm{p} K_{\mathrm{a} 2}^{\mathrm{app}}$ is defined as the $\mathrm{pH}$ value where $\left\langle f_{-1}\right\rangle=\left\langle f_{-2}\right\rangle$. (b) Width of the transition $(\Delta)$ as a function of $D$. The width $\Delta$ is defined as the difference between the $\mathrm{pH}$ values where the degree of dissociation reaches the values of $1 / 11$ and $10 / 11\left(\Delta_{1}\right.$ for $\left\langle f_{-1}\right\rangle$ and $\Delta_{2}$ for $\left.\left\langle f_{-2}\right\rangle\right)$; see text. Horizontal dashed lines show the bulk values for the $\mathrm{p} K_{\mathrm{a}}$ 's and $\Delta$. Horizontal dotted lines show the predictions of the simple analytical model, eqs $20-23$. Parameters: $N_{\mathrm{p}} / A(R)=0.2$ chains $/ \mathrm{nm}^{2}$, degree of polymerization $N=28, \mathrm{p} K_{\mathrm{a} 1}^{\text {bulk }}=4.5, \mathrm{p} K_{\mathrm{a} 2}^{\text {bulk }}=7.7$, and $C_{\text {salt }}=$ $0.1 \mathrm{M}$.

the cylindrical nanochannel on the values of $\mathrm{p} K_{\mathrm{a} 1}^{\mathrm{app}}, \mathrm{p} K_{\mathrm{a} 2}^{\mathrm{app}}$, and the widths of the respective transitions, $\Delta_{1}$ and $\Delta_{2}$. We define the widths of the transitions as the difference between the $\mathrm{pH}$ values where the degree of dissociation is $1 / 11$ and 10/11:

$$
\begin{aligned}
& \Delta_{1}=\mathrm{pH}\left(\left\langle f_{-1}\right\rangle=\frac{10}{11}\right)-\mathrm{pH}\left(\left\langle f_{-1}\right\rangle=\frac{1}{11}\right) \\
& \Delta_{2}=\mathrm{pH}\left(\left\langle f_{-2}=\frac{10}{11}\right\rangle\right)-\mathrm{pH}\left(\left\langle f_{-2}\right\rangle=\frac{1}{11}\right)
\end{aligned}
$$

This definition of $\Delta$ was chosen to ensure $\Delta=2$ for an ideal acid-base equilibrium (see Figure $4 S$ ).

Figure 6 shows that $\mathrm{p} K_{\mathrm{a}}^{\mathrm{app}}$ and $\Delta$ increase for decreasing nanochannel diameter. The $\mathrm{p} K_{\mathrm{a}}^{\mathrm{app}}$ differs from the $\mathrm{p} K_{\mathrm{a}}^{\text {bulk }}$ because the acid-base equilibrium is displaced toward the uncharged segments to minimize the local electrostatic repulsions in the system (a process known as charge regulation), as we explained above. The transition widths for MEP monomers in the bulk are $2.03 \mathrm{pH}$ units for the two chemical equilibria, which are slightly larger than the ideal value of $2 \mathrm{pH}$ units due to the coupling between the two acid-base equilibria in the bulk. This coupling is rather small for MEP because its two $\mathrm{p} K_{\mathrm{a}}$ 's are separated by more than $2 \mathrm{pH}$ units, $\mathrm{p} K_{\mathrm{a} 2}-\mathrm{p} K_{\mathrm{a} 1}=3.2 .^{36}$

To understand the effect of confinement on the chemical transitions, we propose a simple analytical model, which is a simplified formulation of the general molecular theory. This model is useful to understand the shifts of the apparent $\mathrm{p} K_{\mathrm{a}}$ 's and the transition widths $\Delta$ shown in Figure $6 \mathrm{a}$ and $\mathrm{b}$. The model, described in detail in the Supporting Information, considers a homogeneous polymer layer on a flat surface interacting with a homogeneous bulk solution, that is, a twophase system, and assumes $f_{-2}=0$ during the first transition and $f_{0}=0$ during the second transition. The analytical expressions, eqs 17 and 18, for the first and second acid-base equilibria, respectively, require the following input parameters: $f_{-1}, f_{-2}$ (fraction of dissociation of MEP monomers in the film), $\phi_{p}$ (polymer volume fraction in the film, we use the value $\phi_{p}=$ 0.053 for both transitions), and $C_{\text {salt }}$ (salt concentration in the bulk). The apparent $\mathrm{p} K_{\mathrm{a}}$ constants can thus be calculated as the $\mathrm{pH}$ where $f_{-1}=0.5$ (for $\mathrm{p} K_{\mathrm{a} 1}^{\mathrm{app}}$ ) or $f_{-2}=0.5$ (for $\mathrm{p} K_{\mathrm{a} 2}^{\mathrm{app}}$ ).

$$
\begin{array}{r}
\mathrm{pH}\left(f_{-1}\right)=\mathrm{p} K_{\mathrm{a} 1}^{\text {bulk }}+\log _{10}\left(\frac{f_{-1}}{1-f_{-1}}\right) \\
+\log _{10}\left(\frac{1}{0.602 C_{\text {salt }} v_{p}} \frac{f_{-1} \phi_{p}}{\left(1-\phi_{p}\right)^{v_{\mathrm{s}} / v_{\mathrm{w}}}}\right) \\
\mathrm{pH}\left(f_{-2}\right)=\mathrm{p}_{\mathrm{a} 2}^{\text {bulk }}+\log _{10}\left(\frac{f_{-2}}{1-f_{-2}}\right) \\
+\log _{10}\left(\frac{1}{0.602 C_{\text {salt }} v_{p}} \frac{\left(1+f_{-2}\right) \phi_{p}}{\left(1-\phi_{p}\right)^{v_{\mathrm{s}} / v_{\mathrm{w}}}}\right)
\end{array}
$$

The predictions of the simple model (dotted lines in Figure 6a) are in good agreement with the calculations of the molecular theory (symbols in Figure 6a) for large diameters $(D$ $>50 \mathrm{~nm}$ ). This result is important because it shows that it is possible to capture the chemical equilibrium shift with a simple model in the range of large diameters $(D>h)$. As expected, reducing the salt concentration increases the free-energy cost of uncharging a MEP monomer in the film and increases the shift of the apparent $\mathrm{p} K_{\mathrm{a}}$ 's with respect to the bulk values (see Figure S3).

For channel radii smaller than the length of a fully extended polymer chain (10 $\mathrm{nm}$ in our case), the simple model fails to reproduce the predictions of the molecular theory because this model does not account for the effect of the curvature of the surface. In conclusion, for narrow channels, where nanoconfinement shifts the chemical equilibria toward the uncharged states, the molecular details of the polymer layer become important and should be explicitly taken into account.

The expressions for the widths of the protonation transitions in the simple model are (see Supporting Information):

$$
\begin{aligned}
& \Delta_{1}=\Delta^{\text {ideal }}+\log _{10}\left(10 \frac{\phi_{p}^{\mathrm{B}}}{\left(1-\phi_{p}^{\mathrm{B}}\right)^{v_{\mathrm{s}} / v_{\mathrm{w}}}} \frac{\left(1-\phi_{p}^{\mathrm{A}}\right)^{v_{\mathrm{s}} / v_{\mathrm{w}}}}{\phi_{p}^{\mathrm{A}}}\right) \\
& \Delta_{2}=\Delta^{\text {ideal }}+\log _{10}\left(\frac{21}{12}\right)=2.24
\end{aligned}
$$

In eq $22, \Delta^{\text {ideal }}=2$ is the ideal transition width, and $\phi_{p}^{\mathrm{A}}$ and $\phi_{p}^{\mathrm{B}}$ are the volume fraction of the homogeneous polymer layer when $\left\langle f_{-1}\right\rangle=1 / 11$ and $\left\langle f_{-1}\right\rangle=10 / 11$, respectively. Note that $\Delta_{2}$ is independent of $\phi_{p}$ because we assume that the density profile of the polyelectrolyte is unaffected by the second deprotonation step (see Figure $4 \mathrm{a}$ ). In the case of $\Delta_{1}$, we use 
$\phi_{p}^{\mathrm{A}}=0.053$ (see above) and estimate $\phi_{p}^{\mathrm{A}}=0.1$ from the results in Figure 5 (the criteria used in this choice are discussed in Figure S2). Equations 22 and 23 show that the widths of the transitions have an ideal contribution $\Delta^{\text {ideal }}=2$ plus a nonideal part.

Figure $6 \mathrm{~b}$ shows the width of the $0 /-1$ transition $(0)$ and the $-1 /-2$ transition (red $\square$ ) for a salt concentration of $0.1 \mathrm{M}$. The green horizontal dashed line indicates the ideal bulk width of $2.03 \mathrm{pH}$ units, while the red and black dotted lines show the widths predicted by our simple model for the first and second transitions, respectively. For large channel diameters and $C_{\text {salt }}=$ $0.1 \mathrm{M}$, eqs 22 and 23 correctly capture the nonideal contribution to the transition widths. We observe transition widths of 2.67 (first transition) and 2.24 (second transition), which are equal to the widths of the transition of polyelectrolyte brushes on a flat substrate determined with the simple model (dotted line). The simple model also deviates from the predictions of the molecular theory for narrow pores, as was explained above for the apparent equilibrium constants.

As we discussed above, the free-energy cost of protonation of a MEP monomer is larger inside the nanochannel than in the bulk due to the electrostatic repulsions between the negative charges on these monomers. This free-energy cost of protonation depends on the molecular organization of the film, such as the conformation of the polyelectrolyte and the content of counterions. The molecular organization changes continuously as the $\mathrm{pH}$ is varied (see Figures 4 and 5), which results in a continuous change of the protonation free-energy cost, and, therefore, the acid-base transition width becomes larger than the ideal one. In other words, the nonideal contribution to the transition width in eqs 22 and 23 arises from the variation of the electrostatic interactions within the polyelectrolyte film during the protonation process ${ }^{9,42-44}$ (it is possible to think about this effect as an acid-base titration where the effective $\mathrm{pK}_{\mathrm{a}}$ shifts with $\mathrm{pH}$ ). It is also very interesting to note that the width of the second transition is always smaller than the width of the first transition. This result is explained by the fact that the reorganization of the system during the $0 /-1$ transition is more dramatic than that during the $-1 /-2$ transition (see the counterion concentration in Figure 4 and volume fraction profiles for the polyelectrolyte in Figure 5).

Acid-Base Equilibria and Conductance in Noncylindrical Nanochannels. We introduce in this section results for conical and trumped-shaped nanochannels. We computed the molecular properties of conical and trumpet-shaped nanochannel using nanochannel dimensions based on experimental values: ${ }^{45} D_{\min }=10 \mathrm{~nm}$ (tip diameter), $D_{\max }=290 \mathrm{~nm}$ (base diameter), and $L=12 \mu \mathrm{m}$ (channel length).

Figure $7 \mathrm{~b} 1$ and $\mathrm{b} 2$ shows the charge per monomer averaged over the whole nanochannel, $\left\langle Q_{\text {mon }}\right\rangle$, as a function of $\mathrm{pH}$ (the calculation of average values for noncylindrical nanochannels within our theoretical framework is explained in the Supporting Information). The nanochannel conductance (Figure $7 \mathrm{cl}$ and c2) results from the inverse of the total nanochannel resistance given by eq 16. Comparison of the conductance of nanochannels with different shapes but equal base and tip diameters in Figure 7 shows that the trumpet-shaped nanochannel has a lower conductance than the conical nanochannel. This effect arises because for the same tip and base diameters, the trumpetshaped nanochannel has a smaller diameter, and thus a larger resistance, than the conical nanochannel at all intermediate $z$ positions.

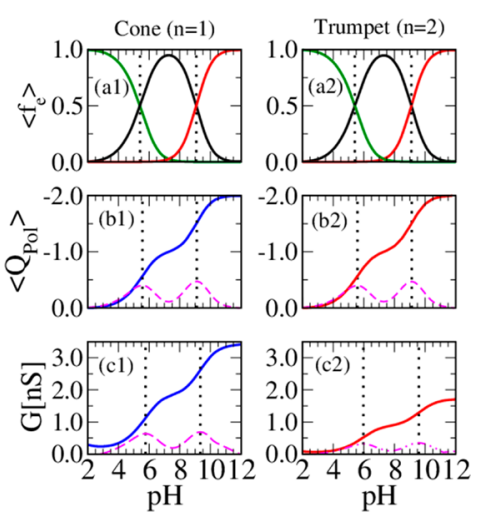

Figure 7. (a1, a2) Average fractions of dissociation, $\left\langle f_{\mathrm{e}}\right\rangle$, as a function of $\mathrm{pH}$ for the three different monomer states, that is, $e=0$ (neutral monomer, green curve), $e=-1$ (black curve), and $e=-2$ (red curve). (b1, b2) Average charge per monomer as a function of $\mathrm{pH}$; the dashed line shows the first derivative with respect to $\mathrm{pH}$. (c) Conductance of the nanochannel, $G$, as a function of $\mathrm{pH}$; the dashed line shows the first derivative of the curve with respect to $\mathrm{pH}$. Dotted lines show the $\mathrm{p} K_{\mathrm{a}}^{\text {app }}$ estimated from $\left\langle f_{0}\right\rangle=\left\langle f_{-1}\right\rangle$ and $\left\langle f_{-1}\right\rangle=\left\langle f_{-2}\right\rangle$ in (a1, a2) and the first derivative of $G$ in $(\mathrm{c} 1, \mathrm{c} 2) . D_{\min }=10 \mathrm{~nm}, D_{\max }=290 \mathrm{~nm}, L=12$ $\mu \mathrm{m}, C_{\text {salt }}=0.1 \mathrm{M}$.

The values of $\mathrm{p} K_{\mathrm{a}}$ cond estimated from the conductance curves and the $\mathrm{pK}_{\mathrm{a}}^{\mathrm{app}}$ directly obtained from the $\left\langle f_{e}\right\rangle$ curves are different for the conical and trumpet nanochannels. Table 1 compiles the values of $\mathrm{p} K_{\mathrm{a}}^{\mathrm{app}}, \mathrm{p} K_{\mathrm{a}}^{\text {cond }}$, and $\Delta \mathrm{p} K_{\mathrm{a} i}^{\mathrm{app}}=\mathrm{p} K_{\mathrm{a} i}^{\text {cond }}-$ $\mathrm{p} K_{\mathrm{a} i}^{\mathrm{app}}$, for $i=1,2$ and the different nanochannel shapes.

Table 1. Values of Apparent $\mathrm{p} K_{\mathrm{a}}$ 's Determined from the Speciation Curves ( $\left.\mathrm{p} K_{\mathrm{a} i}{ }^{\mathrm{app}}\right)$, Inflection Point of the Conductance versus $\mathrm{pH}$ Curves $\left(\mathrm{pK}_{\mathrm{a} i}{ }^{\text {cond }}\right)$, and Difference between These Values $\Delta \mathrm{p} K_{\mathrm{a} i}{ }^{\mathrm{app}}$, for the First $(i=1)$ and Second $(i=2)$ Transitions and Different Channel Shapes

\begin{tabular}{lccc} 
& cylinder & cone & trumpet \\
$\mathrm{p} K_{\mathrm{a} 1}{ }^{\text {app }}$ & 5.81 & 5.40 & 5.41 \\
$\mathrm{p} K_{\mathrm{a} 1}{ }^{\text {cond }}$ & 6.06 & 5.76 & 5.97 \\
$\Delta \mathrm{p} K_{\mathrm{a} 1}^{\text {app }}$ & 0.25 & 0.36 & 0.56 \\
$\mathrm{p} K_{\mathrm{a} 2}^{\text {app }}$ & 9.67 & 9.08 & 9.10 \\
$\mathrm{p} K_{\mathrm{a} 2}{ }^{\text {cond }}$ & 9.69 & 9.34 & 9.59 \\
$\Delta \mathrm{p} K_{\mathrm{a} 2}^{\text {app }}$ & 0.02 & 0.26 & 0.49 \\
\hline
\end{tabular}

The results in Table 1 show that the geometry of the pore changes slightly the agreement between the apparent $\mathrm{p} K_{\mathrm{a}}$ obtained from the macroscopic calculated conductance $\left(\mathrm{p} K_{\mathrm{a} i}^{\text {cond }}\right.$ ) and that directly obtained from the protonation state of the system, $\mathrm{p} K_{\mathrm{a} i}^{\mathrm{app}}$. This agreement is important for the experimental characterization of nanochannels of different shapes. The slight disagreement between $\mathrm{pK}_{\mathrm{a}}^{\text {app }}$ and $\mathrm{p} K_{\mathrm{a}}^{\text {cond }}$ in noncylindrical channels is due to the fact that the conductance is more affected by the state of protonation of the polyelectrolytes in the tip of the nanochannel than by those close to the nanochannel base. Therefore, the conductance does not exactly reflect the global state of charge for noncylindrical channels. Table 1 also shows that the apparent $\mathrm{p} K_{\mathrm{a}}$ 's for the trumpet-shape channel are larger (i.e., more shifted with respect to the bulk $\mathrm{p} K_{\mathrm{a}}$ 's) than those for the cone. This result is explained by the fact that the trumpet is narrower than the cone for all $z$, and, as we showed in Figure 6a, the apparent $\mathrm{p} K_{\mathrm{a}}$ 's increase for decreasing channel radii. 


\section{CONCLUSIONS}

In this work, we introduced a new theoretical approach to calculate the low-bias conductance of polyelectrolyte-modified nanochannels of arbitrary shape. Our methodology is based on approximating the total resistance of noncylindrical nanochannels as the series combination of the resistances of cylindrical slices of different diameters, which can be calculated using a molecular theory. This approximation, which is expected to be valid for long aspect ratios (length larger than radius), was validated against a full solution of the NernstPlanck-Poisson equations using finite elements for nanochannels with constant surface charge. The theoretical approach introduced in this work complements previous approaches to study polyelectrolyte-modified channels and pores, which were restricted either to long cylindrical nanochannels $^{28}$ or to short nanopores. ${ }^{46}$ We have also introduced a simplification of the general molecular theory approach to study the shifts in the apparent $\mathrm{p} K_{\mathrm{a}}$ of the polyelectrolyte due to nanoconfinement. This model neglects the curvature of the surface and thus describes the shift of $\mathrm{pK}$ and width of transitions when diameters are much larger than the thickness of the polymer layer.

We applied our theory to study the acid-base properties of diprotic polyacids, which is interesting due to the presence of two acid-base transitions. We showed that as the $\mathrm{pH}$ rises and the polyelectrolyte increases its charge, three main mechanisms emerge to reduce the electrostatic repulsions: (i) shifting the chemical equilibrium, (ii) stretching polymer conformations, and (iii) changing the concentration of free ions inside the nanochannel. All of these mechanisms are combined to minimize the total free energy of the system. Decreasing the channel diameter shifts the chemical equilibrium to the uncharged state (i.e., it favors the first regulation mechanism) and broadens the two acid-base transitions of PMEP. We also show that the first deprotonation process has a broader transition width than the second for all diameters.

We addressed the question whether the inflection point of the conductance versus $\mathrm{pH}$ curve can be used to estimate the apparent $\mathrm{p} K_{\mathrm{a}}$ of the weak polyelectrolyte. We found that for a cylindrical nanochannel, the apparent equilibrium constant obtained from the calculated conductance versus $\mathrm{pH}$ data is in good agreement with the $\mathrm{p} K_{\mathrm{a}}^{\mathrm{app}}$ obtained directly from the state of protonation of the monomers. For other nanochannel geometries, the agreement between the two methods of calculating the apparent $\mathrm{p} K_{\mathrm{a}}$ slightly worsens and can be as large as one-half $\mathrm{pH}$ unit, due to the fact that the state of charge in the narrow regions of the channel has a bigger effect on the conductance than that in the wide parts. These results are important as they establish the accuracy of using conductance versus $\mathrm{pH}$ data to estimate the protonation state of weak acidbase species within single chemically modified nanochannels.

\section{ASSOCIATED CONTENT}

\section{S Supporting Information}

The Supporting Information is available free of charge on the ACS Publications website at DOI: 10.1021/acs.jpcc.5b11788.

Description of the simple model (homogeneous polymer layer), comparison of experimental conductance versus $\mathrm{pH}$ data, and comparison between the conductance predicted by our theory and a full finite elements calculation for a nanochannel with surface charges (PDF)

\section{AUTHOR INFORMATION}

\section{Corresponding Authors}

*E-mail: mario@qi.fcen.uba.ar.

*E-mail: azzaroni@inifta.unlp.edu.ar.

\section{Notes}

The authors declare no competing financial interest.

\section{ACKNOWLEDGMENTS}

F.M.G. acknowledges a doctoral scholarship from CONICET (Argentina). O.A. and M.T. are CONICET fellows. F.M.G. would like to thank R. Nap and G. Longo for stimulating discussions and G. Putzel for valuable comments and criticisms. This work was supported as part of the Center for Bio-Inspired Energy Science, an Energy Frontier Research Center funded by the U.S. Department of Energy, Office of Science, Basic Energy Sciences, under award no. DE-SC0000989. This research was supported in part through the computational resources and staff contributions provided by the Quest high performance computing facility at Northwestern University, which is jointly supported by the Office of the Provost, the Office for Research, and Northwestern University Information Technology. O.A. acknowledges financial support from ANPCyT (PICT 20102554 and PICT-2013-0905), Consejo Nacional de Investigaciones Científicas y Técnicas (CONICET) (PIP 11220130100370CO), Fundación Petruzza, and the Austrian Institute of Technology GmbH (AIT-CONICET Partner Lab: "Exploratory Research for Advanced Technologies in Supramolecular Materials Science” - Exp. 4947/11, res. no. 3911).

\section{REFERENCES}

(1) Wen, L.; Hou, X.; Tian, Y.; Zhai, J.; Jiang, L. Bio-Inspired Photoelectric Conversion Based on Smart-Gating Nanochannels. Adv. Funct. Mater. 2010, 20 (16), 2636-2642.

(2) Dekker, C. Solid-State Nanopores. Nat. Nanotechnol. 2007, 2, 209-215.

(3) Kowalczyk, S. W.; Blosser, T. R; Dekker, C. Biomimetic Nanopores: Learning from and about Nature. Trends Biotechnol. 2011, 29 (12), 607-614.

(4) Howorka, S.; Siwy, Z. S. Nanopores as Protein Sensors. Nat. Biotechnol. 2012, 30 (6), 506-507.

(5) Martin, C. R.; Siwy, Z. S. Chemistry. Learning Nature's Way: Biosensing with Synthetic Nanopores. Science 2007, 317 (5836), 331332.

(6) Hoogerheide, D. P.; Garaj, S.; Golovchenko, J. a. Probing Surface Charge Fluctuations with Solid-State Nanopores. Phys. Rev. Lett. 2009, 102 (25), 256804.

(7) Schoch, R.; Han, J.; Renaud, P. Transport Phenomena in Nanofluidics. Rev. Mod. Phys. 2008, 80 (3), 839-883.

(8) Guo, W.; Xia, H.; Xia, F.; Hou, X.; Cao, L.; Wang, L.; Xue, J.; Zhang, G.; Song, Y.; Zhu, D.; et al. Current Rectification in Temperature-Responsive Single Nanopores. ChemPhysChem 2010, 11 (4), 859-864.

(9) Gillespie, D.; Eisenberg, R. Modified Donnan Potentials for Ion Transport through Biological Ion Channels. Phys. Rev. E: Stat. Phys., Plasmas, Fluids, Relat. Interdiscip. Top. 2001, 63 (6), 061902.

(10) Guo, W.; Tian, Y.; Jiang, L. Asymmetric Ion Transport through Ion-Channel-Mimetic Solid-State Nanopores. Acc. Chem. Res. 2013, 46 (12), 2834-2846.

(11) Siwy, Z. S.; Apel, P.; Baur, D.; Dobrev, D. Preparation of Synthetic Nanopores with Transport Properties Analogous to Biological Channels. Surf. Sci. 2003, 532-535, 1061-1066.

(12) Ram, P.; Apel, P. Y.; Cervera, J.; Maf, S.; Ramírez, P.; Mafé, S.; Apel, P. Y.; Cervera, J.; Mafé, S. Pore Structure and Function of Synthetic Nanopores with Fixed Charges: Tip Shape and Rectification Properties. Nanotechnology 2008, 19 (31), 315707. 
(13) Siwy, Z. S. Ion-Current Rectification in Nanopores and Nanotubes with Broken Symmetry. Adv. Funct. Mater. 2006, 16 (6), $735-746$.

(14) Stein, D.; Kruithof, M.; Dekker, C. Surface-Charge-Governed Ion Transport in Nanofluidic Channels. Phys. Rev. Lett. 2004, 93 (3), 035901.

(15) Ali, M.; Schiedt, B.; Healy, K.; Neumann, R.; Ensinger, W. Modifying the Surface Charge of Single Track-Etched Conical Nanopores in Polyimide. Nanotechnology 2008, 19 (8), 085713.

(16) Siwy, Z. S.; Heins, E.; Harrell, C. C.; Kohli, P.; Martin, C. R. Conical-Nanotube Ion-Current Rectifiers: The Role of Surface Charge. J. Am. Chem. Soc. 2004, 126 (35), 10850-10851.

(17) van der Heyden, F. H. J.; Bonthuis, D. J.; Stein, D.; Meyer, C.; Dekker, C. Power Generation by Pressure-Driven Transport of Ions in Nanofluidic Channels. Nano Lett. 2007, 7 (4), 1022-1025.

(18) Daiguji, H.; Yang, P.; Szeri, A. J.; Majumdar, A. Electrochemomechanical Energy Conversion in Nanofluidic Channels. Nano Lett. 2004, 4 (12), 2315-2321.

(19) Mafe, S.; Manzanares, J. a.; Ramirez, P. Gating of Nanopores: Modeling and Implementation of Logic Gates. J. Phys. Chem. C 2010, 114 (49), 21287-21290.

(20) Ali, M.; Mafe, S.; Ramirez, P.; Neumann, R.; Ensinger, W. Logic Gates Using Nanofluidic Diodes Based on Conical Nanopores Functionalized with Polyprotic Acid Chains. Langmuir 2009, 25 (20), 11993-11997.

(21) Daiguji, H.; Oka, Y.; Shirono, K. Nanofluidic Diode and Bipolar Transistor. Nano Lett. 2005, 5 (11), 2274-2280.

(22) Venkatesan, B. M. B.; Bashir, R. Nanopore Sensors for Nucleic Acid Analysis. Nat. Nanotechnol. 2011, 6 (10), 615-624.

(23) Siwy, Z. S.; Trofin, L.; Kohli, P.; Baker, L. a; Trautmann, C.; Martin, C. R. Protein Biosensors Based on Biofunctionalized Conical Gold Nanotubes. J. Am. Chem. Soc. 2005, 127 (14), 5000-5001.

(24) Ali, M.; Neumann, R; Ensinger, W. ACS Nano 2010, 4 (12), $7267-7274$

(25) Ali, M.; Nasir, S.; Ramirez, P.; Cervera, J.; Mafe, S.; Ensinger, W. Carbohydrate-Mediated Biomolecular Recognition and Gating of Synthetic Ion Channels. J. Phys. Chem. C 2013, 117 (35), 1823418242.

(26) Ali, M.; Yameen, B.; Neumann, R.; Ensinger, W.; Knoll, W.; Azzaroni, O. Biosensing and Supramolecular Bioconjugation in Single Conical Polymer Nanochannels. Facile Incorporation of Biorecognition Elements into Nanoconfined Geometries. J. Am. Chem. Soc. 2008, 130 (48), 16351-16357.

(27) Ali, M.; Ramirez, P.; Tahir, M. N.; Mafe, S.; Siwy, Z.; Neumann, R.; Tremel, W.; Ensinger, W. Biomolecular Conjugation inside Synthetic Polymer Nanopores via Glycoprotein-Lectin Interactions. Nanoscale 2011, 3 (4), 1894-1903.

(28) Tagliazucchi, M.; Azzaroni, O.; Szleifer, I. Responsive Polymers End-Tethered in Solid-State Nanochannels: When Nanoconfinement Really Matters. J. Am. Chem. Soc. 2010, 132 (35), 12404-12411.

(29) Brunsen, A.; Díaz, C.; Pietrasanta, L. I.; Yameen, B.; Ceolín, M.; Soler-Illia, G. J. a a; Azzaroni, O. Proton and Calcium-Gated Ionic Mesochannels: Phosphate-Bearing Polymer Brushes Hosted in Mesoporous Thin Films as Biomimetic Interfacial Architectures. Langmuir 2012, 28 (7), 3583-3592.

(30) Wang, D.; Nap, R. J.; Lagzi, I.; Kowalczyk, B.; Han, S.; Grzybowski, B. a; Szleifer, I. How and Why Nanoparticle's Curvature Regulates the Apparent pKa of the Coating Ligands. J. Am. Chem. Soc. 2011, 133 (7), 2192-2197.

(31) Nap, R.; Gong, P.; Szleifer, I. Weak Polyelectrolytes Tethered to Surfaces: Effect of Geometry, Acid-base Equilibrium and Electrical Permittivity. J. Polym. Sci., Part B: Polym. Phys. 2006, 44, 2638-2662.

(32) Dong, R.; Lindau, M.; Ober, C. K. Dissociation Behavior of Weak Polyelectrolyte Brushes on a Planar Surface. Langmuir 2009, 25 (8), 4774-4779.

(33) Szleifer, I.; Carignano, M. A.; Lafayette, W. Tethered Polymer Layers; Wiley: New York, 1996; Vol. XC.
(34) Nap, R. J.; Tagliazucchi, M.; Szleifer, I. Born Energy, Acid-Base Equilibrium, Structure and Interactions of End-Grafted Weak Polyelectrolyte Layers. J. Chem. Phys. 2014, 140 (2), 024910.

(35) Tagliazucchi, M.; de la Cruz, M. O.; Szleifer, I. Self-Organization of Grafted Polyelectrolyte Layers via the Coupling of Chemical Equilibrium and Physical Interactions. Proc. Natl. Acad. Sci. U. S. A. 2010, 107 (12), 5300-5305.

(36) Skoog, D. A.; West, D. M. W.; Holler, F. J.; Crouch, S. R. C. Fundamentals of Analytical Chemistry, 7th ed.; Saunders College Pub.: Philadelphia, PA, 1995.

(37) Tagliazucchi, M.; Rabin, Y.; Szleifer, I. Ion Transport and Molecular Organization Are Coupled in Polyelectrolyte-Modified Nanopores. J. Am. Chem. Soc. 2011, 133 (44), 17753-17763.

(38) Goldman, D. Potential, Impedance, and Rectification in Membranes. J. Gen. Physiol. 1943, 27, 1.

(39) Yameen, B.; Ali, M.; Neumann, R.; Ensinger, W.; Knoll, W.; Azzaroni, O. Proton-Regulated Rectified Ionic Transport through Solid-State Conical Nanopores Modified with Phosphate-Bearing Polymer Brushes. Chem. Commun. (Cambridge, U. K.) 2010, 46 (11), $1908-1910$

(40) Tagliazucchi, M.; Rabin, Y.; Szleifer, I. Transport Rectification in Nanopores with Outer Membranes Modified with Surface Charges and Polyelectrolytes. ACS Nano 2013, 7 (10), 9085-9097.

(41) Tagliazucchi, M.; Szleifer, I. Stimuli-Responsive Polymers Grafted to Nanopores and Other Nano-Curved Surfaces: Structure, Chemical Equilibrium and Transport. Soft Matter 2012, 8 (28), 7292.

(42) Ohshima, H.; Ohki, S. Donnan Potential and Surface Potential of a Charged Membrane. Biophys. J. 1985, 47 (5), 673-678.

(43) Wang, T.-Y.; Sheng, Y.-J.; Tsao, H.-K. Donnan Potential of Dilute Colloidal Dispersions: Monte Carlo Simulations. J. Colloid Interface Sci. 2009, 340 (2), 192-201.

(44) Higa, M.; Tanioka, A.; Kira, A. A Novel Measurement Method of Donnan Potential at an Interface between a Charged Membrane and Mixed Salt Solution. J. Membr. Sci. 1998, 140 (2), 213-220.

(45) Yameen, B.; Ali, M.; Neumann, R.; Ensinger, W.; Knoll, W.; Azzaroni, O. Ionic Transport through Single Solid-State Nanopores Controlled with Thermally Nanoactuated Macromolecular Gates. Small 2009, 5 (11), 1287-1291.

(46) Tagliazucchi, M.; Rabin, Y.; Szleifer, I. Ion Transport and Molecular Organization Are Coupled in Polyelectrolyte-Modified Nanopores. J. Am. Chem. Soc. 2011, 133 (44), 17753-17763. 